\title{
Three-Dimensional Tracking of Interfacial Hopping Diffusion
}

\author{
Dapeng Wang, ${ }^{1,2}$ Haichao Wu, ${ }^{2}$ and Daniel K. Schwartz, ${ }^{2, *}$ \\ ${ }^{1}$ State Key Laboratory of Polymer Physics and Chemistry, Changchun Institute of Applied Chemistry, \\ Chinese Academy of Sciences, Changchun 130022, People's Republic of China \\ ${ }^{2}$ Department of Chemical and Biological Engineering, University of Colorado Boulder, Boulder, Colorado 80309, USA
}

(Received 13 September 2017; published 29 December 2017)

\begin{abstract}
Theoretical predictions have suggested that molecular motion at interfaces-which influences processes including heterogeneous catalysis, (bio)chemical sensing, lubrication and adhesion, and nanomaterial self-assembly — may be dominated by hypothetical "hops" through the adjacent liquid phase, where a diffusing molecule readsorbs after a given hop according to a probabilistic "sticking coefficient." Here, we use three-dimensional (3D) single-molecule tracking to explicitly visualize this process for human serum albumin at solid-liquid interfaces that exert varying electrostatic interactions on the biomacromolecule. Following desorption from the interface, a molecule experiences multiple unproductive surface encounters before readsorption. An average of approximately seven surface collisions is required for the repulsive surfaces, decreasing to approximately two and a half for surfaces that are more attractive. The hops themselves are also influenced by long-range interactions, with increased electrostatic repulsion causing hops of longer duration and distance. These findings explicitly demonstrate that interfacial diffusion is dominated by biased 3D Brownian motion involving bulk-surface coupling and that it can be controlled by influencing short- and long-range adsorbate-surface interactions.
\end{abstract}

DOI: 10.1103/PhysRevLett.119.268001

Molecular transport in fluid phases is understood in terms of the Brownian motion of individual molecules and particles, and can, therefore, be predicted and controlled by parameters including the hydrodynamic radius and fluid viscosity. In contrast, the analogous behavior at the interfaces remains poorly understood despite its fundamental interest and technological relevance; i.e., the dynamics of macromolecules at solid-liquid interfaces underlie many applications including chemical sensing, catalysis, lubrication, and adhesion [1-6]. While interfacial diffusion is nominally two dimensional (2D) and conventionally described in terms of 2D Brownian motion, longstanding theoretical models [7-16] have predicted that interfacial mass transport could actually be dominated by "flights" through an adjacent liquid phase, which would dramatically alter the nature of interfacial molecular motion; an understanding of this process is necessary in order to rationally control mass transport at surfaces. Recent experimental results indirectly support these predictions by measuring the $2 \mathrm{D}$ projection of trajectories for atoms, molecules, polymers, and nanoparticles, in thin films, at solid-liquid interface, and on lipid bilayers, which can be represented as an intermittent process with periods of apparent immobility alternating with long flights comprising a heavy-tailed distribution [17-26]. However, the evidence for the presence of three-dimensional (3D) hops remains indirect, and critical aspects of the proposed "hopping" process remain a mystery. For example, theoretical models represent a flight as a series of hops (i.e., returns to the surface), where a molecule may stochastically readsorb after a given hop according to a hypothetical parameter known as the "sticking coefficient" [27]. While this parameter is theoretically the primary determinant of the flight length, to date there has been no actual support for the presence of multiple hops per flight. Moreover, efforts to model the putative 3D hops have necessarily involved the simplistic assumption of unbiased 3D Brownian motion. While this assumption may be reasonable in the absence of knowledge to the contrary, it is likely not correct in the presence of long-range molecule-surface interactions. To understand desorption-mediated surface transport and searching [3,28-31], and to exploit it for useful ends, it is desirable to directly visualize the flight process itself, including the nature of any 3D component of the motion and the surface capture and release process, which requires $3 \mathrm{D}$ tracking capabilities.

To directly test and quantitatively characterize the putative 3D nature of surface diffusion, we employed a 3D single-molecule imaging approach that combined variable-angle illumination epifluorescence microscopy with double-helix point spread function (DH PSF) optics (SPINDLE ${ }^{\mathrm{TM}}$ module, Double Helix LLC, Boulder) with a high-efficiency phase mask [see Fig. 1(a); further details are described in the Supplemental Material [32] ]. The fully 3D tracking capability allowed us to comprehensively address fundamental questions regarding macromolecular diffusion at solid-liquid interfaces. Most importantly, we verified the theoretical prediction that surface diffusion is, in fact, dominated by 3D flights. Moreover, the direct observation of hopping provided a comprehensive and detailed picture of the ways in which short- and longrange adsorbate-surface interactions influence interfacial 
(a)
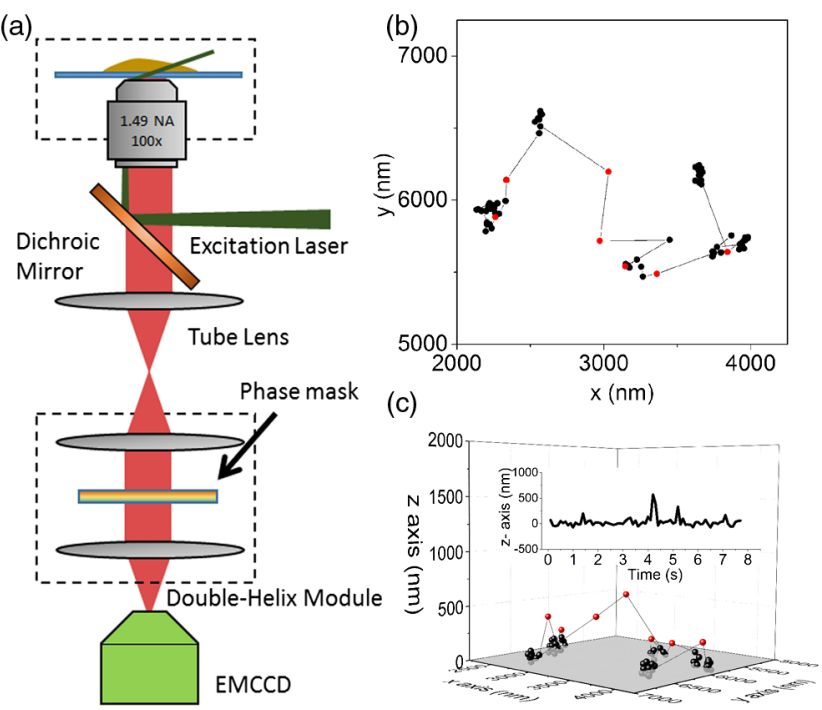

FIG. 1. (a) A standard wide-field microscope body was equipped with a Double-Helix SPINDLE module to implement DH PSF imaging. (b) Two-dimensional projection of a trajectory. (c) Actual 3D motion for the trajectory shown in (b). To guide the eye, the red spheres indicate steps for the polymer in solution while the black ones denote steps at the surface. The inset shows the corresponding trace of $z$ position vs time.

dynamics by controlling waiting times between flights, the temporal duration of hops, the 3D spatial extent of hops, and the probability of readsorption after a hop.

The tracer molecules used in this study were human serum albumin (HSA) labeled with Alexa 555. Since the isoelectric point of unlabeled HSA is $p \mathrm{H} 4.7$, the molecules exhibited a net negative charge at the neutral $p \mathrm{H}$ employed here. To make the motion of HSA compatible with the spatiotemporal resolution of the tracking method [37], it was dispersed in a water-glycerol mixture (7:93; nominal viscosity $367 \mathrm{cP})$ at concentrations in the range $10^{-14}-10^{-12} \mathrm{M}$, resulting in a sufficiently sparse surface coverage $\left(<0.04\right.$ molecule $\left./ \mu \mathrm{m}^{2}\right)$ to facilitate single-molecule localization. To study the effects of electrostatic interactions, we used negatively charged fused silica (FS) and FS modified by various mixtures of a "nonfouling" and nonionic oligo (ethylene glycol) silane and a positively charged amino-silane $\left(\mathrm{NH}_{2}\right)$ functionality (denoted as $\mathrm{NH}_{2}-\mathrm{X} \%$, where $\mathrm{X}$ reflects the fraction of $\mathrm{NH}_{2}$ groups). Thus, the strongest electrostatic repulsion was between the HSA and unmodified FS. The repulsion was decreased and eventually changed to attraction with increasing $\mathrm{NH}_{2}$ surface concentrations. The surface modification procedure is detailed in the Supplemental Material [32].

The DH PSF was created using a newly designed phase mask placed in the Fourier plane of the image train of a Nikon Ti-Eclipse microscope [Fig. 1(a)]. Further details of the technique can be found in the Supplemental Material [32]. DH PSF imaging permitted direct observations of 3D desorption-mediated hops and flights, explicitly confirming this mechanism of mass transport. As indicated by the representative trajectory displayed in Figs. 1(b) and 1(c), the 3D trajectory elucidates the detailed nature of hops (denoted by red symbols), which are not revealed in a 2D surface projection. By directly observing and analyzing many such trajectories, we found that trajectories generally included many unproductive surface encounters [Figs. 2(a), 2(b), and Fig. S4 in Ref. [32] ], countering the conventional view.

We quantified the surface dynamics by calculating the ensemble-average mean-squared displacement (MSD) via $\Delta R(\tau)^{2}=\left\langle|R(t+\tau)-R(t)|^{2}\right\rangle$, where $t$ denotes the molecules at time $t, \tau$ indicates the lag time, and $R$ is the $3 \mathrm{D}$ position. Figure 2(c) shows plots of MSD vs $\tau$ for HSA on surfaces of varying adsorbate-surface interactions. As shown in Fig. S5 of Ref. [32], this 3D MSD was dominated by in-plane motion, while the MSD in the $z$ direction exhibited strong apparent confinement effects due to the limited axial tracking distance. Interestingly, the apparent diffusion coefficient (proportional to the slope of the MSD) depended strongly on the surface charge, with HSA diffusing most rapidly on the repulsive FS surfaces and systematically more slowly as protein-surface interactions became more attractive. An apparent short-time diffusion coefficient $D_{\text {app }}$ was obtained via fitting $\Delta R(\tau)^{2}=6 D_{\text {app }} \tau$ over the first four data points, yielding values of 0.083 ,

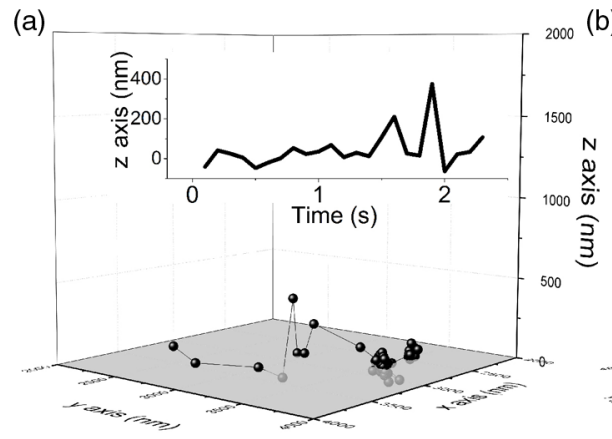

(b)
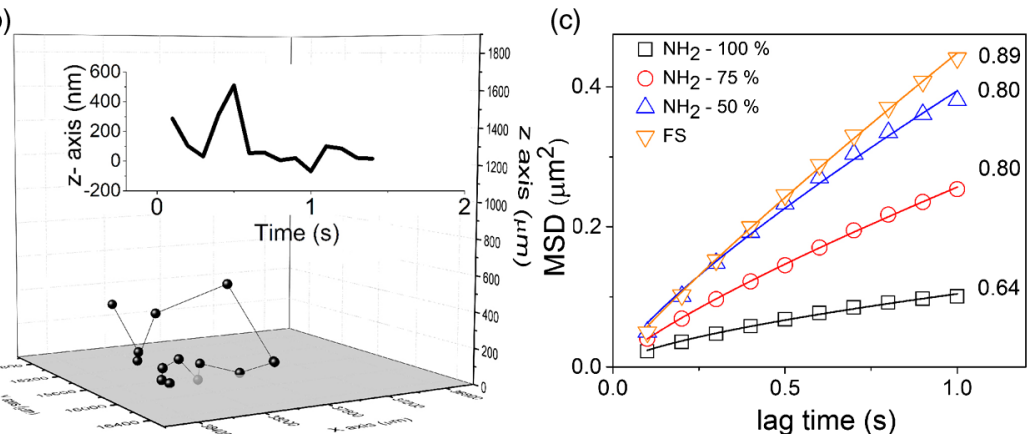

FIG. 2. (a),(b) Representative trajectories for HSA on a $\mathrm{NH}_{2}$-modified FS surface. The inset shows the corresponding trace of $z$ position vs time. (c) Mean-squared displacement vs lag time for HSA on surfaces with varying adsorbate-surface interactions. The subdiffusive scaling exponents are annotated on the right. 
$0.079,0.050$, and $0.025 \mu \mathrm{m}^{2} / \mathrm{s}$ on the FS, $\mathrm{NH}_{2}-50 \%$, $\mathrm{NH}_{2}-75 \%$, and $\mathrm{NH}_{2}-100 \%$ surfaces, respectively. For longer times, the plots of MSD vs $\tau$ were not linear but instead indicated subdiffusive behavior [Fig. 2(c)], exhibiting a power-law dependence on $\tau$ with an exponent smaller than unity. The deviation from linearity was weakest for the repulsive FS surface (with an exponent of 0.90), and the behavior became increasingly subdiffusive for surfaces that had strong attractive interactions with HSA; the exponent decreased to 0.64 on the $\mathrm{NH}_{2}-100 \%$ surfaces (Table S2 in Ref. [32]). Thus, the surface diffusion was fast and nearly Brownian for surfaces from which the macromolecule experienced electrostatic repulsion and became dramatically slower and more subdiffusive for more attractive surfaces. These ensemble-averaged statistical trends can presumably be explained microscopically by the details of intermittent trajectories [18], e.g., waiting times, hop distances, and sticking coefficients, which can, in turn, be directly related to molecular-level interactions.

The analysis of 3D trajectories allowed us to determine the statistics of the desorption-mediated hops in detail. Uniquely, the sticking coefficient was calculated by counting the fraction of all surface encounters that resulted in actual adsorption. To distinguish between adsorption and unproductive surface encounters in the presence of localization uncertainty, we applied a criterion for adsorption that included both lateral mobility and $z$ position; a detailed description is given in the Supplemental Material [32]. Figure 3 shows the apparent sticking coefficient on surfaces with varying adsorbate-surface interactions. Interestingly, the adsorption probability was significantly smaller than unity in all cases, indicating that a diffusing molecule typically encountered and sampled the surface many times prior to adsorption. Interestingly, we found that the apparent sticking coefficient depended systematically on the adsorbate-surface interactions ranging from a maximum of $0.45 \pm 0.03$ for the most attractive $\mathrm{NH}_{2}-100 \%$ surface (i.e., $>2$ encounters per adsorption) to a minimum of $0.13 \pm 0.01$ on the repulsive FS surface (approximately eight encounters per adsorption event). We use the term "apparent" sticking coefficient because there are presumably many small and fast displacements away from the surface that cannot be resolved, resulting in underestimation of the absolute number of hops per flight. However,

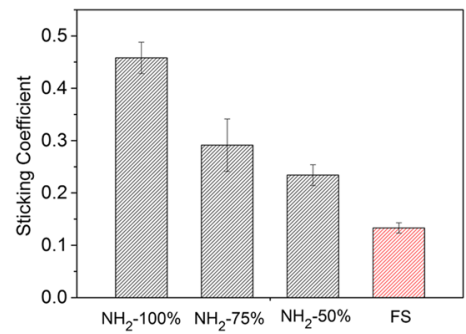

FIG. 3. Apparent sticking coefficient of HSA on different surfaces. this does not affect the systematic trends associated with electrostatic interactions described here. Further discussion of this issue is provided in the Supplemental Material [32].

Long-range adsorbate-surface interactions also influenced the hop length, hop height, and hop duration, suggesting that the near-surface 3D diffusion was not simply Brownian. As shown in Fig. 4(a), for the more repulsive $\mathrm{FS}$ and $\mathrm{NH}_{2}-50 \%$ surfaces, the distributions of hop length (i.e., the lateral distance between successive surface encounters during flights) exhibited a heavy tail of very long hops $(\geq 0.4 \mu \mathrm{m})$, which was dramatically depressed for the more attractive $\mathrm{NH}_{2}-100 \%$ surface. Interestingly, we found that the heavy tails in the hoplength distributions could be asymptotically described as a power law, $\sim 1 / r^{\beta}$ (as shown in Fig. S6 in Ref. [32]), with exponents in the range 2.8-3.4, in agreement with the theoretically calculated Cauchy tail $P \sim 1 / r^{3}$ found previously for desorption-mediated diffusion [8]. This consistency indicates that the 3D measurement correctly captures the underlying mechanism of desorption-mediated surface dynamics. The trend in the hop-length distribution with surface charge was hypothesized to be due to the fact that long-range electrostatic interactions influenced the return time, defined as the time between consecutive surface encounters. For example, if surface repulsion reduced the likelihood of motion towards the surface, an adsorbate molecule would exhibit flights of longer duration and distance. Further supporting this hypothesis, the distributions of height during hops (see Fig. S7 in Ref. [32]) were apparently influenced by electrostatic interactions, with hops on FS extending farther from the surface compared to hops on $\mathrm{NH}_{2}-100 \%$ surfaces.

To quantitatively test this hypothesis, we calculated the return time using only trajectories comprising multiple encounters in a given flight; the cumulative return-time probability distributions are shown in Fig. 4(b). These distributions clearly indicate that the return process was strongly influenced by surface interactions, where returns were faster with increasing positive surface charge (attraction) and slower when the surface was negatively charged (repulsion). Combined with the trends exhibited by the hop-length distributions [Fig. 4(a)], the return-time data suggest that the near-surface diffusion of macromolecules experiences a bias associated with long-range electrostatic interactions. This effect was enhanced in these experiments because the solvent was chosen to maximize the range of electrostatic interactions (i.e., the salt-free glycerol-water mixture had a calculated Debye length of $\sim 780 \mathrm{~nm}$ ). Combined, these data suggest that for the $\mathrm{NH}_{2}-100 \%$ surface, electrostatic attraction between HSA and $\mathrm{NH}_{2}$ "pulls" the object back to the surface more quickly than expected for unbiased Brownian motion (causing short hop lengths), and that for the FS surface, electrostatic repulsion "pushes" a molecular away from the surface, causing longer hop lengths. 


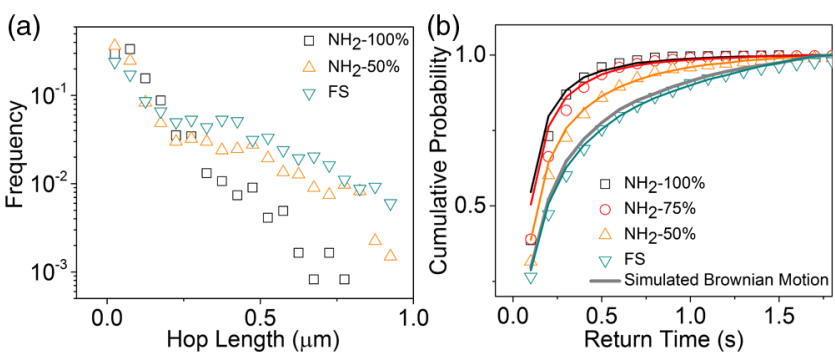

FIG. 4. (a) Hop-length distributions during flights for HSA on surfaces with varying surface-adsorbate interactions. (b) The cumulative probability of return time between consecutive surface encounters. The symbols denote the experimental data on different surfaces, and the solid lines indicate the simulation results described in the main text. The gray line in (b) represents the result of simulated Brownian motion.

In order to test this hypothesis quantitatively, we modeled the return process using kinetic Monte Carlo simulations involving biased Brownian motion in the surface-normal direction [38]. The simulation details are described in the Supplemental Material [32]. In short, generalized random walk simulations were performed on a 1D lattice where the probabilities of steps in the positive or negative direction were biased by the presence of electrostatic interactions. In particular, the probability of moving towards the surface was given as $P=0.5+A e^{-z / L}$ where $L$ is the calculated Debye length, $z$ is the distance to the surface, and $A$ is a parameter corresponding to the direction and magnitude of the bias. These simulations had minimal assumptions and employed experimentally measured diffusion coefficients (see the Supplemental Material [32]) as inputs; the only adjustable parameter was $A$. The model reduced to Brownian motion in the limit of $A=0$, while $A= \pm 0.5$ indicated deterministic 1D directional motion. The values of $A<0$ and $A>0$ corresponded to biased Brownian motion away from and towards the surface, respectively.

By setting $A=0$, we simulated trajectories that exhibited unbiased Brownian motion and measured the distribution of return times (retaining only trajectories that returned within $2 \mathrm{~s}$ to mimic the limitations of experimental tracking). As shown in Fig. 4(b) (gray line), we found that $90 \%$ of the Brownian trajectories returned to the surface within $1 \mathrm{~s}$. This indicated that even unbiased Brownian motion can effectively mediate lateral surface diffusion, in contrast with the intuitive notion that desorbed molecules may return irreversibly to bulk solution. More generally, the return-time distribution was sensitive to the bias parameter $A$. By systematically varying $A$, the simulations successfully represented the experimental return-time distributions at different surfaces. The values of $A$ that best described our data for attractive surfaces were $0.17,0.30$, and 0.35 for the $\mathrm{NH}_{2}-50 \%$, $\mathrm{NH}_{2}-75 \%$, and $\mathrm{NH}_{2}-100 \%$ surfaces, respectively. On the other hand, a slightly repulsive value of $A=-0.04$ represented the return process of HSA on the negatively charged FS surface. Taken together, the consistent systematic trends seen in both simulations and experimental results suggest that biased Brownian motion due to long-range electrostatic interactions between HSA and surfaces can quantitatively describe the desorption-mediated near-surface dynamics in the surface-normal direction.

A final important component of the intermittent diffusion process involves the duration of waiting times $\tau_{\text {des }}$ between flights. Previous 2D studies have shown that apparent waiting time distributions $\Psi\left(\tau_{\text {des }}\right)$ often had heavy tails (asymptotically described by power laws) and were correlated with the strength of short-range interactions [39]. Here, we observed similar phenomena using 3D tracking measurements. For all surfaces, the distribution was described by a power-law relation $\Psi\left(\tau_{\text {des }}\right) \sim \tau_{\text {des }}^{-(1+\alpha)}$ in the range $0.5 \mathrm{~s}<\tau_{\text {des }}<5 \mathrm{~s}$ (Fig. 5). The parameter $\alpha$ (annotated in Fig. 5) provided an empirical way to correlate the statistics of $\Psi\left(\tau_{\mathrm{des}}\right)$ with adsorbate-surface interactions. As the adsorbate-surface interaction was varied from repulsive (FS surfaces) to attractive ( $\mathrm{NH}_{2}-100 \%$ surfaces), $\alpha$ decreased systematically, denoting that waiting times were longer (i.e., the distributions were broader) on surfaces corresponding to a stronger adsorbate-surface interaction.

Previous work has suggested that surface diffusion was influenced by multiple effects, e.g., adsorbate-surface interactions (desorption rate), readsorption probability, physical obstacles, chemical surface heterogeneity, etc. [24,39-43]. The results presented here provide a comprehensive picture of how and why adsorbate-surface interactions influence interfacial motion. In particular, we found a clear and dramatic overall trend in the apparent diffusion coefficient [Fig. 2(c)], where molecules diffused rapidly in the presence of electrostatic repulsion and slowed dramatically as electrostatic attraction increased. A detailed analysis of 3D trajectories shows that this trend was the cumulative effect of multiple mechanisms: as electrostatic attraction increased, waiting times lengthened, individual hops became shorter in duration and distance, and a molecule was more likely to readsorb after each hop. While molecular-level effects were not explicitly considered here (i.e., HSA molecules were treated essentially as a model charged particle to focus on the

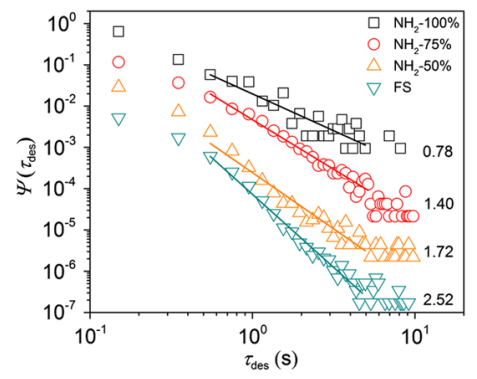

FIG. 5. Distribution of waiting times between flights for HSA on different surfaces. The symbols denote the experimental data, and the solid lines indicate power-law fits in the range of $0.5-5 \mu \mathrm{m}$. The scaling exponents $\alpha$ obtained using an equation described in the main text are annotated on the right. 
general hopping phenomena), it is likely that the detailed microscopic mechanisms of adsorption and desorption may be coupled to intramolecular phenomena such as conformational changes [44]. In our analysis, the effects of such changes on surface dynamics were embedded in kinetic parameters such as waiting times, hop lengths, return time, and so on.

Desorption-mediated transport has important implications for the efficiency and rate of molecular "searching," e.g., for interfacial reactive and/or binding sites [30,31, 45-47]. Indeed, a recent experimental study found that the improvement of surface searching efficiency was even greater than that predicted by a simple intermittent hopping model, especially at long distances [47]. The results presented here provide a potential explanation for these observations. When a molecule desorbs, it diffuses through solution but still samples the surface via electrostatic interactions. Moreover, even under strongly adsorbing conditions, a molecule encounters the surface many times before final readsorption. These factors may explain why the actual searching efficiency is greater than predicted by theoretical models where flights are assumed to be knowledge-free cruises, during which the molecule obtains no information about the surface [45-47].

In summary, a 3D tracking method was used to study the effect of electrostatic interactions on the desorptionmediated 3D flights of a negatively charged tracer molecule at solid-liquid interfaces of varying surface charge. Once a molecule desorbed, it experienced multiple unproductive surface encounters before readsorption. Therefore, the sticking coefficient, i.e., the adsorption probability, was much less than unity even at a strongly attractive surface and decreased further as the adsorbate-surface interaction (attraction) decreased. Moreover, the near-surface dynamics were quantitatively consistent with biased Brownian motion (associated with long-range electrostatic interactions), leading to strong bulk-surface coupling. An important consequence of this picture is that the surface searching is more efficient than predicted by conventional theory. In general, biased Brownian motion can have significant physical implications for interfacial adsorption, transport, sensing, self-assembly, and searching phenomena that are related to the near-surface dynamics.

This work was supported by the U.S. Department of Energy, Office of Science, Basic Energy Sciences, under Award No. DE-SC0001854. D. W. is thankful for support from Chinese "The Thousand Talents Plan" for Young Professionals and International Science and Technology Cooperation Program of Jilin, China.

*Corresponding author. daniel.schwartz@colorado.edu

[1] P.-G. De Gennes, Scaling Concepts in Polymer Physics (Cornell University Press, Ithaca, 1979).
[2] S. A. Sukhishvili, Y. Chen, J. D. Muller, E. Gratton, K. S. Schweizer, and S. Granick, Nature (London) 406, 146 (2000).

[3] B. O'Shaughnessy and D. Vavylonis, J. Phys. Condens. Matter 17, R63 (2005).

[4] I. M. Zaid, M. A. Lomholt, and R. Metzler, Biophys. J. 97, 710 (2009).

[5] L. Xu, V. Kozlovskaya, E. Kharlampieva, J. F. Ankner, and S. A. Sukhishvili, ACS Macro Lett. 1, 127 (2012).

[6] V. V. Palyulin, A. V. Chechkin, and R. Metzler, Proc. Natl. Acad. Sci. U.S.A. 111, 2931 (2014).

[7] O. V. Bychuk and B. O’Shaughnessy, J. Chem. Phys. 101, 772 (1994).

[8] O. V. Bychuk and B. O’Shaughnessy, Phys. Rev. Lett. 74, 1795 (1995).

[9] R. Metzler and J. Klafter, Phys. Rep. 339, 1 (2000).

[10] J. A. Revelli, C. E. Budde, D. Prato, and H. S. Wio, New J. Phys. 7, 16 (2005).

[11] M. A. Lomholt, K. Tal, R. Metzler, and K. Joseph, Proc. Natl. Acad. Sci. U.S.A. 105, 11055 (2008).

[12] A. V. Chechkin, I. M. Zaid, M. A. Lomholt, I. M. Sokolov, and R. Metzler, J. Chem. Phys. 134, 204116 (2011).

[13] S. Burov, J. H. Jeon, R. Metzler, and E. Barkai, Phys. Chem. Chem. Phys. 13, 1800 (2011).

[14] J. Klafter and I. M. Sokolov, First Steps in Random Walks: From Tools to Applications (Oxford University Press, New York, 2011).

[15] A. V. Chechkin, I. M. Zaid, M. A. Lomholt, I. M. Sokolov, and R. Metzler, Phys. Rev. E 86, 041101 (2012).

[16] F. Rojo, C. E. Budde, Jr., H. S. Wio, and C. E. Budde, Phys. Rev. E 87, 012115 (2013).

[17] M. Schunack, T. R. Linderoth, F. Rosei, E. Lægsgaard, I. Stensgaard, and F. Besenbacher, Phys. Rev. Lett. 88, 156102 (2002).

[18] M. J. Skaug, J. Mabry, and D. K. Schwartz, Phys. Rev. Lett. 110, 256101 (2013).

[19] C. Yu, J. Guan, K. Chen, S. C. Bae, and S. Granick, ACS Nano 7, 9735 (2013).

[20] L. J. Tauzin, B. Shuang, L. Kisley, A. P. Mansur, J. Chen, A. de Leon, R. C. Advincula, and C. F. Landes, Langmuir 30, 8391 (2014).

[21] X. Sun, S. Xiao, H. Deng, and W. Hu, Chem. Phys. Lett. 634, 108 (2015).

[22] L. Wei, Z. Ye, Y. Xu, B. Chen, E. S. Yeung, and L. Xiao, Anal. Chem. 88, 11973 (2016).

[23] S. W. Chee, Z. Baraissov, N. D. Loh, P. T. Matsudaira, and U. Mirsaidov, J. Phys. Chem. C 120, 20462 (2016).

[24] D. Wang, H.-Y. Chin, C. He, M. P. Stoykovich, and D. K. Schwartz, ACS Macro Lett. 5, 509 (2016).

[25] K. Shezad, K. Zhang, M. Hussain, H. Dong, C. He, X. Gong, X. Xie, J. Zhu, and L. Shen, Langmuir 32, 8238 (2016).

[26] D. Krapf, G. Campagnola, K. Nepal, and O. B. Peersen, Phys. Chem. Chem. Phys. 18, 12633 (2016).

[27] J. Mabry and D. K. Schwartz, J. Phys. Chem. Lett. 6, 2065 (2015).

[28] G. M. Viswanathan, S. V. Buldyrev, S. Havlin, M. Da Luz, E. Raposo, and H. E. Stanley, Nature (London) 401, 911 (1999)

[29] O. Bénichou, C. Loverdo, M. Moreau, and R. Voituriez, Phys. Rev. E 74, 020102 (2006). 
[30] S. Condamin, O. Bénichou, and J. Klafter, Phys. Rev. Lett. 98, 250602 (2007).

[31] O. Bénichou, D. Grebenkov, P. Levitz, C. Loverdo, and R. Voituriez, Phys. Rev. Lett. 105, 150606 (2010).

[32] See the Supplemental Material at http://link.aps.org/ supplemental/10.1103/PhysRevLett.119.268001, which includes Refs. [33-36], for details of the sample preparation, technique description, analysis methods, and simulation procedure.

[33] S. R. P. Pavani, M. A. Thompson, J. S. Biteen, S. J. Lord, N. Liu, R. J. Twieg, R. Piestun, and W. E. Moerner, Proc. Natl. Acad. Sci. U.S.A. 106, 2995 (2009).

[34] G. Grover, K. DeLuca, S. Quirin, J. DeLuca, and R. Piestun, Opt. Express 20, 26681 (2012).

[35] D. Wang, A. Agrawal, R. Piestun, and D. K. Schwartz, Appl. Phys. Lett. 110, 211107 (2017).

[36] J.-P. Hansen and I. R. McDonald, Theory of Simple Liquids (Elsevier, New York, 1990).

[37] H. Shen, L. J. Tauzin, R. Baiyasi, W. Wang, N. Moringo, B. Shuang, and C. F. Landes, Chem. Rev. 117, 7331 (2017).
[38] A. M. Berezhkovskii, L. Dagdug, and S. M. Bezrukov, J. Chem. Phys. 147, 014103 (2017).

[39] N. Nelson and D. K. Schwartz, J. Phys. Chem. Lett. 4, 4064 (2013).

[40] Q. Yang and J. Zhao, Langmuir 27, 11757 (2011).

[41] D. Wang, C. He, M. P. Stoykovich, and D. K. Schwartz, ACS Nano 9, 1656 (2015).

[42] J. N. Mabry and D. K. Schwartz, J. Phys. Chem. Lett. 6, 2065 (2015).

[43] D. Giri, K. M. Ashraf, M. M. Collinson, and D. A. Higgins, J. Phys. Chem. C 119, 9418 (2015).

[44] P. Vilaseca, K. A. Dawson, and G. Franzese, Soft Matter 9, 6978 (2013).

[45] O. Bénichou, C. Loverdo, M. Moreau, and R. Voituriez, Phys. Chem. Chem. Phys. 10, 7059 (2008).

[46] O. Bénichou, C. Loverdo, M. Moreau, and R. Voituriez, Rev. Mod. Phys. 83, 81 (2011).

[47] J. H. Monserud and D. K. Schwartz, Phys. Rev. Lett. 116, 098303 (2016). 